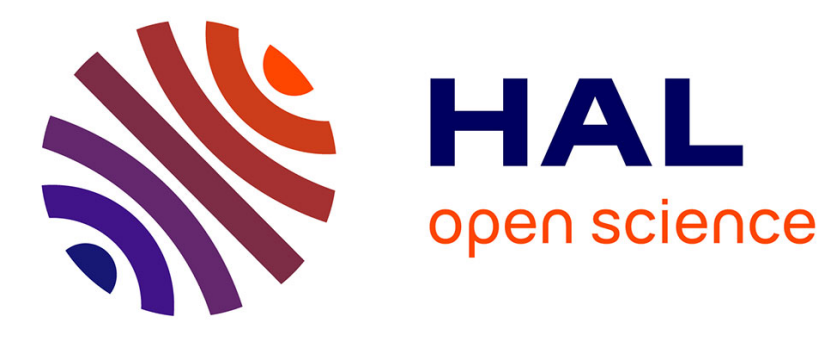

\title{
miR-323a regulates ERBB4 and is involved in depression.
}

Laura M Fiori, Aron Kos, Rixing Lin, Jean-Francois Théroux, Juan Pablo

Lopez, Claudia Kühne, Carola Eggert, Maria Holzapfel, Rosa-Eva Huettl, Naguib Mechawar, et al.

\section{To cite this version:}

Laura M Fiori, Aron Kos, Rixing Lin, Jean-Francois Théroux, Juan Pablo Lopez, et al.. miR-323a regulates ERBB4 and is involved in depression.. Molecular Psychiatry, 2020, 10.1038/s41380-02000953-7 . hal-03019942

\section{HAL Id: hal-03019942 https://hal.science/hal-03019942}

Submitted on 23 Nov 2020

HAL is a multi-disciplinary open access archive for the deposit and dissemination of scientific research documents, whether they are published or not. The documents may come from teaching and research institutions in France or abroad, or from public or private research centers.
L'archive ouverte pluridisciplinaire HAL, est destinée au dépôt et à la diffusion de documents scientifiques de niveau recherche, publiés ou non, émanant des établissements d'enseignement et de recherche français ou étrangers, des laboratoires publics ou privés. 


\section{miR-323a regulates ERBB4 and is involved in depression}

Laura M. Fiori PhD ${ }^{1,}{ }^{*}$, Aron Kos PhD ${ }^{2,3}{ }^{*}$, Rixing Lin BSc ${ }^{1}$, Jean-Francois Théroux MSc ${ }^{1}$, Juan Pablo Lopez PhD ${ }^{2,3}$, Claudia Kühne DVM ${ }^{2}$, Carola Eggert ${ }^{2}$, Maria Holzapfel ${ }^{2}$, Rosa-Eva Huettl PhD ${ }^{2}$, Naguib Mechawar PhD ${ }^{1}$,

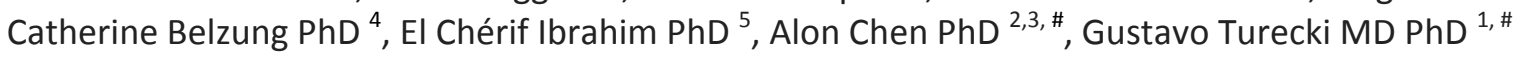

${ }^{1}$ Douglas Mental Health University Institute, Department of Psychiatry, McGill University, Montreal, Canada.

${ }^{2}$ Max Planck Institute of Psychiatry, Department of Stress Neurobiology and Neurogenetics, Munich, Germany.

${ }^{3}$ Weizmann Institute of Science, Department of Neurobiology, Rehovot, Israel.

${ }^{4}$ UMR 1253, iBrain, UFR Sciences et Techniques, Parc Grandmont, Tours, France.

${ }^{5}$ Aix-Marseille Université, CNRS, INT, Institute Neuroscience Timone, Marseille, France.

* These authors contributed equally

\# Co-corresponding authors

\section{Corresponding Author:}

Gustavo Turecki, MD PhD

McGill Group for Suicide Studies

Douglas Mental Health University Institute

Frank B Common Pavilion

Room F-3125

6875 LaSalle Boulevard

Montreal, Quebec, H4H 1R3

Email: gustavo.turecki@mcgill.ca

Phone: (514) 761-6131 Ext: 2369

Fax: (514) 762-3023

Running Title: miR-323a and MDD 


\section{Abstract:}

Major depressive disorder (MDD) is a complex and debilitating illness whose etiology remains unclear. Small RNA molecules, such as micro RNAs (miRNAs) have been implicated in MDD, where they display differential expression in the brain and the periphery. In this study, we quantified miRNA expression by small RNA sequencing in the anterior cingulate cortex and habenula of individuals with MDD and psychiatrically-healthy controls. Thirty-two miRNAs showed significantly correlated expression between the two regions (False Discovery Rate < 0.05), of which four, miR-204-5p, miR-320b, miR-323a-3p, and miR-331-3p, displayed upregulated expression in MDD. We assessed the expression of predicted target genes of differentially expressed miRNAs in the brain, and found that the expression of erb-b2 receptor tyrosine kinase 4 (ERBB4), a gene encoding a neuregulin receptor, was downregulated in both regions, and was influenced by miR-323a-3p in vitro. Finally, we assessed the effects of manipulating miRNA expression in the mouse ACC on anxiety- and depressive-like behaviors. Mice in which miR-323-3p was overexpressed or knocked-down displayed increased and decreased emotionality, respectively. Additionally, these mice displayed significantly downregulated and upregulated expression of Erbb4, respectively. Overall, our findings indicate the importance of brain miRNAs in the pathology of MDD, and emphasize the involvement of miR-323a-3p and ERBB4 in this phenotype. Future studies further characterizing miR-323a-3p and neuregulin signaling in depression are warranted. 
Major depressive disorder (MDD) is a prevalent and debilitating illness, and is a leading cause of premature death worldwide ${ }^{1}$. While its etiology and pathology remain unclear, various neurobiological factors have been robustly associated with MDD, many of which are the target of common pharmacological treatments. Recent studies have highlighted the role of small RNA molecules in MDD and other psychiatric conditions ${ }^{2-4}$. Among these, the most well-studied are microRNAs (miRNAs), which are single-stranded RNA molecules 17- to 22- nucleotides (nt) in length. MiRNAs play essential roles in regulating gene expression through translational silencing and degradation of target mRNA molecules through interactions with the 3' untranslated region (UTR) of the target mRNA and a complementary region of the miRNA (seed region) ${ }^{5}$. Additionally, one miRNA can regulate the expression of multiple genes, allowing each miRNA to influence numerous signaling pathways ${ }^{6}$. The expression patterns of miRNAs vary according to tissue, and many demonstrate enriched and region-specific expression in the brain ${ }^{7}$, where miRNAs play a key role in a number of cellular processes such as neurogenesis and synapse development ${ }^{8,9}$, as well as learning and memory ${ }^{10}$. In additional to normal brain processes, a number of studies have now identified differential expression of miRNAs in psychiatric disorders, including MDD ${ }^{2}$, bipolar disorder ${ }^{3}$, and schizophrenia ${ }^{4}$.

Numerous candidate miRNA studies have been performed in brain tissue of depressed individuals or animal models of depression, and have highlighted miRNAs involved in various pathways including the polyamine system, brain-derived neurotrophic factor signaling, and monoaminergic neurotransmission ${ }^{11}$. A few transcriptome-wide studies in humans have investigated the prefrontal cortex ${ }^{2,12-14}$, and the locus coeruleus ${ }^{15}$ of depressed suicides. In this study, we investigate transcriptome-wide miRNA expression in the dorsal anterior cingulate cortex (ACC) and the lateral habenula of individuals who died during an episode of MDD, and psychiatrically-healthy controls. Numerous studies have implicated the ACC in MDD, and have identified altered gene expression ${ }^{16}$, as well as changes in volume, which appear to be related to treatment response ${ }^{17,18}$. In addition, it is the preferred site for deep brain stimulation (DBS) in treatment-resistant depression ${ }^{19}$. The lateral habenula has also been implicated in depression through neuroimaging and molecular studies, and is composed of neurons that are activated by negative emotions ${ }^{20}$. Similar to the ACC, clinical and preclinical studies have also linked this region to antidepressant response ${ }^{21,22}$.

In this study, we identified four miRNAs, miR-204-5p, miR-320b, miR-323a-3p, and miR-331-3p, which displayed differential brain expression in MDD. Additionally, the expression of putative mRNA targets of these miRNAs were found to be differentially expressed in the brain, and influenced by miR-323a-3p in vitro. Furthermore, we modified the expression of miR-323a in the mouse ACC, and found it to be associated with both anxiety- and depression-like behavioral phenotypes. 
miR-323a and MDD

Materials and Methods:

\section{Human Studies:}

\section{Human Samples:}

Post-mortem samples of dorsal ACC (Brodmann Area 24) and lateral habenula were obtained, in collaboration with the Quebec Coroner's Office, from the Douglas-Bell Canada Brain Bank (Douglas Mental Health University Institute, Montreal, Quebec, Canada). We analysed a total of 117 samples, comprised of 80 ACC samples, and 37 lateral habenula samples, as detailed in Supplemental Table 1. Groups were matched for post-mortem interval (PMI), $\mathrm{pH}$ and age. Psychological autopsies were performed as described previously, based on DSM-IV criteria ${ }^{23}$. The control group had no history of major psychiatric disorders. All cases met criteria for MDD or depressive disorder not-otherwise-specified. Written informed consent was obtained from next-of-kin. This study was approved by the Douglas Hospital Research Centre institutional review board.

\section{Small RNA sequencing and analysis:}

RNA was extracted from all brain samples using a combination of the miRNeasy Mini kit and the RNeasy MinElute Cleanup kit (Qiagen), with DNase treatment, and divided into small (<200nt) and large (>200nt) fractions. RNA quality, represented as RNA Integrity Number (RIN), was assessed using the Agilent 2200 Tapestation. Small RNA-seq libraries were prepared from the small RNA fraction, using the Illumina TruSeq Small RNA protocol following the manufacturer's instructions. Samples were sequenced at the McGill University and Genome Quebec Innovation Centre (Montreal, Canada) using the Illumina HiSeq2000 with 50nt single-end reads. All sequencing data were processed using CASAVA 1.8+ (Illumina) and extracted from FASTQ files. The Fastx_toolkit was used to trim the Illumina adapter sequences. Additional filtering based on defined cutoffs was applied, including: 1) Phred quality (Q) mean scores higher than 30,2) reads between 15-40nt in length, 3) adapter detection based on perfect-10nt match, and 4) removal of reads without detected adapter. Additionally, we used Bowtie ${ }^{24}$ to align reads to the human genome (GRCh37) and ncPRO-seq ${ }^{25}$ in combination with miRBase (V20) to match them to known miRNA sequences ${ }^{26}$. Furthermore, all sequencing data was normalized with the Bioconductor - DESeq2 package ${ }^{27}$, using a detection threshold of 10 counts per miRNA. Processing of miRNA reads was performed for ACC and habenula samples separately. We retained all miRNAs with $>10$ reads in $70 \%$ of either group (controls, cases) for differential analyses. Quality metrics are shown in Supplemental Table 2. RNA extractions, sequencing, and data processing were conducted by blinded investigators.

\section{Real-time PCR (RT-PCR):}

Human: For measurement of miRNAs in human samples, RNA from the small RNA fraction was reverse transcribed using pre-designed TaqMan RT-PCR miRNA assays (Applied Biosystems) according to the manufacturer's instructions. RNU6B or U6 were used as endogenous controls. For measurement of mRNA expression, RNA from the large RNA fraction was reverse-transcribed using M-MLV Reverse Transcriptase (200 $\mathrm{U} / \mathrm{uL}$ ) (ThermoFisher) with a combination of oligo (dT) and random primers. RT-PCR was performed using SYBR green (Applied Biosystems) with GAPDH as an endogenous control. Reactions for both miRNAs and mRNAs were run in triplicate using the QuantStudio 6 Flex System and data collected using QuantStudio Real-Time PCR Software v1.1. Expression levels were calculated using the absolute (standard curve method) or relative $\left(2^{-\Delta \Delta C t}\right)$ quantification method, depending on experimental design. Primer sequences for mRNAs are shown in Supplemental Table 3.

Mouse: For measuring mature mouse miR-323-3p (equivalent to human miR-323a-3p), cDNA was generated using the TaqMan MicroRNA Reverse Transcription Kit (Applied Biosystems). RT-PCR was performed using the TaqMan Universal Master Mix II, no UNG kit (Applied Biosystems). cDNA synthesis and RT-PCR were performed according to the manufacturer's instructions. The following gene-specific Taqman assays were used: miR-323- 
miR-323a and MDD

$3 p$ assay ID 002227, U6 snRNA assay ID 001973, using U6 as endogenous normalization gene. For measuring Erbb4, cDNA was generated using the high-capacity cDNA RT kit with RNase inhibitor (Applied Biosystems). RTPCR was performed using QuantiFast SYBR green PCR Kit (Qiagen). Both CDNA and RT-PCR were performed according to the manufacturer's instructions. Rpl13a was used as endogenous normalization gene. For both miRNA and mRNA, RT-PCR data was collected on the QuantStudio 7 Flex Real-Time PCR System (Applied Biosystems). Relative miR-323-3p and Erbb4 expression differences were calculated by applying the delta $\mathrm{Ct}$ method. Primer sequences for mRNAs are shown in Supplemental Table 3.

\section{Targeted gene analysis:}

We used five target prediction algorithms, miRWalk 2.0, miRanda, RNA22, RNAhybrid, and Targetscan ${ }^{28-32}$, to identify putative targets of miRNAs. Although miRNAs can bind to other regions of mRNAs, we restricted our searches to the 3' UTR of target mRNAs.

\section{Mouse Studies:}

\section{Animal housing and care:}

Male CD-1 (ICR) were bred and housed in specific-pathogen-free (SPF), temperature-controlled $\left(23 \pm 1^{\circ} \mathrm{C}\right)$, constant humidity $(55 \pm 10 \%)$ and 12-hour light/dark cycle conditions. The age of the mice ranged from 4-7 months old. Animals had ad libidum access to food and water. Animals were housed in groups of four. All animal experiments were evaluated and approved by the local commission for the Care and Use of Laboratory Animals of the Government of Upper Bavaria, Germany. No sample size estimate was performed prior to conducting experiments, however the number of animals was based on what is typically used within the field for the behavior tests performed.

\section{Cloning:}

The miR-323 gene sequence was obtained from the Ensembl genome browser database (Ensembl gene ID ENSMUSG00000065617). The pre-miR-323 sequence was taken including 70 nucleotides up- and down-stream. The miR-scrambled control sequence was previously described ${ }^{33}$. Both the pre-miR-323 and pre-miR-scramble sequence were placed $5^{\prime}$ of the eGFP gene. The miR-323-3p sponge and miR-control sponge consist of a concatemer of six binding sites in the $3^{\prime}$ UTR of the eGFP gene with each binding site separated by an ATCG linker sequence. The miR-323 sponge sequence is based on the complementary sequence of mature miR-323$3 p$. The control sponge sequence was based on C. elegans miR-67, of which the mature sequence has minimal sequence identity with human or rodent miRNAs. Each binding site contains an internal bulge to facilitate miR$323-3 p$ binding without leading to degradation of the sponge sequence ${ }^{34}$. The pAAV-EF1a-Cre was used as backbone, purchased from Addgene (Addgene plasmid \#55636; http://n2t.net/addgene:55636). The pre-miRscramble-eGFP, pre-miR-323-eGFP, eGFP inserts were ordered as gBlock gene fragments (IDT), and cloned into the pAAV-EF1a-Cre backbone. The pAAV-EF1a-Cre backbone was cut with BamH1 and EcoR1 (NEB) to remove the Cre gene and the gene fragments were inserted using Gibson Assembly (NEB) according to the manufacturer's protocol. This generated the pAAV-EF1a-pre-scrambled-GFP, pAAV-EF1a-pre-miR323-GFP, PAAV-EF1a-eGFP plasmids. The miR-sponge inserts were ordered as Ultramer single stranded DNA oligos with the Asc1 and EcoR1 restriction sites at the $5^{\prime}$ and $3^{\prime}$ ends respectively (IDT). The single stranded Ultramer oligonucleotides were annealed according to the manufacturer's instructions generating sticky ends. The eGFP gBlock fragment was designed to contain the Asc1 restriction site at the $3^{\prime}$ end. The pAAV-EF1a-eGFP plasmid was linearized by cutting with Asc1 and EcoR1 (NEB). The annealed miR-control-sponge and miR-323-sponge inserts were ligated into the 3'UTR of the eGFP gene using T4 DNA Ligase according to the provided protocol (NEB). This generated the final pAAV-EF1a-control-sponge and pAAV-EF1a-miR323-sponge plasmids. All plasmids were sequenced to check for mutations.

\section{Validation of constructs:}


For validation of the AAV constructs, mouse neuroblastoma neuro2a (N2a) cells were used. These were from an in-house stock, which were not authenticated or verified for mycoplasma contamination. N2a cells were maintained at $37^{\circ} \mathrm{C}$ with $5 \% \mathrm{CO}_{2}$ in Minimum Essential Medium (MEM), 1x Glutamax, supplemented with $1 \mathrm{x}$ non-essential amino acids, $1 \mathrm{mM}$ sodium pyruvate, $100 \mathrm{U} / \mathrm{ml}$ penicillin, $100 \mu \mathrm{g} / \mathrm{ml}$ streptomycin and $10 \%$ fetal bovine serum (FBS, Gibco). For transfection, cells were detached using trypsin and transfected using Screenfect A (ScreenFect $\mathrm{GmbH}$ ) according to the manufacturer's protocol. Total RNA was extracted using the miRNeasy Mini Kit (Qiagen). For validation of the miR-323-3p binding capacity of the miR-323 sponge, 30nM miR-323-3p or non-targeting control miScript miRNA Mimic (Qiagen) were co-transfected with the miR-323 sponge or miRcontrol sponge. Cells were fixed with 4\% PFA-PBS solution and embedded with Fluoromount-G mounting medium containing DAPI (SouthernBiotech). Cells were imaged using a LSM800 confocal microscope (Zeiss). The total number of cells per image was quantified using ImageJ particle detection based on DAPI nuclei staining. The amount of total GFP signal was determined using ImageJ, followed by normalization to the total number of cells.

\section{Virus production:}

Human embryonic kidney cells (HEK293T) were transfected with the gene transfer rAAV plasmid combined with the helper plasmids in an equal molar ratio of $1: 1: 1$ using $1 \mathrm{mg} / \mathrm{ml}$ linear polyethylenimine hydrochloride (PEI). The rAAV (serotype 1/2) particles were harvested three days after transfection by lysing the cells with three consecutive freeze-and-thaw cycles using an ethanol on dry ice bath and $37^{\circ} \mathrm{C}$ water bath. Lysates were centrifuged (3000 rff) followed by purification of the rAAV particles using a Heparin Agarose Type I chromatography column (Sigma). The eluted rAAV particles were PBS washed using a 100000 MWCO Amicon Ultra Filter (Millipore) and suspended in a final volume of $100 \mu l$. The number of viral genomic particles was determined using qPCR resulting in the following titers; AAV1/2-EF1a-Pre-scrambled-GFP 4,9 $10^{11}$ genome

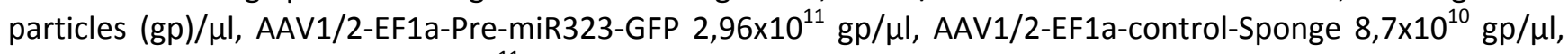
AAV1/2-EF1a-miR323-Sponge $2 \times 10^{11} \mathrm{gp} / \mu \mathrm{l}$.

\section{Stereotactic surgery:}

Eight week-old mice were anesthetized with isoflurane and placed in the stereotactic apparatus (TSE Systems) on a $37^{\circ} \mathrm{C}$ heating pad. Pre-surgery, mice were given Novalgin $(200 \mathrm{mg} / \mathrm{kg}$ body weight) and Metacam (subcutaneous $0.5 \mathrm{mg} / \mathrm{kg}$ body weight). During surgery, mice were continuously supplied with $2 \% \mathrm{v} / \mathrm{v}$ isoflurane in $\mathrm{O}_{2}$ through inhalation. Viruses were injected bilaterally using a 33-gauge injection needle with a $5 \mu$ Hamilton syringe coupled to an automated microinjection pump (World Precision Instruments). $0.25 \mu$ virus was delivered at a rate of $0.1 \mu \mathrm{l} / \mathrm{min}$. The injection coordinates were determined using the Franklin and Paxinos mouse brain atlas, from bregma: $\mathrm{ML}+/-0.3 \mathrm{~mm}$ bilateral; $\mathrm{AP}+1.2 \mathrm{~mm}$; DV $-1.8 \mathrm{~mm}$. After injection the needle was retracted $0.01 \mathrm{~mm}$ and kept at the site for 2.5 minutes, followed by slow withdrawal. After surgery, the animals received Metacam for the 3 following days (intraperitoneal $0.5 \mathrm{mg} / \mathrm{kg}$ body weight). Mice were tested five months after surgery. After completion of the experiments, mice were sacrificed by isoflurane overdose. Brains were removed and fixed in 4\% PFA-PBS followed by dehydration in $30 \%$ sucrose-PBS solution for at least 24 hours each. Brains were sectioned $(50 \mu \mathrm{m})$ using a vibratome (HM $650 \mathrm{~V}$, Thermo Scientific). Brain slices were imaged using the VS120-S6-W slide scanner microscope (Olympus). Injection sites were verified based on GFP expression. The animals were randomly allocated to treatment groups and randomly processed.

\section{Behavioral tests:}

All behavioral tests were conducted by a blinded investigator.

Open field (OF) test: The OF test was performed in a $50 \times 50 \mathrm{~cm}$ light grey box, evenly illuminated with low light conditions (<10 lux). Mice were placed in the open field facing one of the walls and recorded for 5 minutes. Animals were tracked using ANY-maze software (Stoelting). Total distance traveled was used as measure of 
miR-323a and MDD

animal locomotion. Relative time spent in the corners (10 x $10 \mathrm{~cm}$ quadrants in the corners) and the centre (30 $x 30 \mathrm{~cm}$ central section) of the arena were used as measures of anxiety.

Elevated plus maze (EPM): EPM apparatus was made of light grey material, it consists of four intersecting arms elevated approximately $30 \mathrm{~cm}$ above the floor. The two opposing open $(27.5 \times 5 \mathrm{~cm})$ and closed arms $(27.5 \times 5$ $\times 20 \mathrm{~cm})$ are connected with a central zone $(5 \times 5 \mathrm{~cm})$. The animals were placed in the center of the EPM, facing one of the open arms and recorded for 5 minutes under low light conditions ( $<10$ lux). Recordings were analyzed with the ANYmaze software. Relative to control mice the time spent in the open, closed and central areas was calculated and used as anxiety measure.

Tail suspension test (TST): In the TST, animals were taped by their tales on a metal rod, approximately $30 \mathrm{~cm}$ above the ground in a brightly lit room (500 lux). Animals were recorded for 5 minutes, mobility time was quantified using ANY-maze software. Relative mobility time was used as a measure of a depression-like emotional state.

Z-scoring: To combine the data of multiple behavioral tests the z-score of each individual behavioral measure was calculated and integrated as previously described ${ }^{35}$. Briefly, the z-score of each individual behavioral parameter was calculated.

$$
z=\frac{X-\mu}{\sigma}
$$

Data collected from the OF, EPM and TST were combined to calculate an integrated emotionality score.

$$
\text { Emotion z score }=\frac{\mathrm{Z}_{\mathrm{OF}}+Z_{\mathrm{EPM}}+Z_{\mathrm{TST}}}{\text { Number of tests }}
$$

A higher score indicates higher emotional state, while a lower score means a lower emotional state.

\section{Cell Culture:}

Human embryonic kidney cells (HEK293) were purchased from ATCC (CRL-1573) and verified to be free of mycoplasma contamination. Cells were cultured in Dulbecco's Modified Eagle Medium (DMEM) supplemented with $10 \% \mathrm{FBS}, 100 \mathrm{U} / \mathrm{ml}$ penicillin and $100 \mathrm{\mu g} / \mathrm{ml}$ streptomycin (Invitrogen) in a $5 \% \mathrm{CO}_{2}$ humidified incubator at $37^{\circ} \mathrm{C}$.

\section{Endogenous ERBB4 expression:}

For miRNA mimic experiments, cells were treated with 20nM mimic or control (AllStar siRNA) using HiPerfect transfection reagent (Qiagen). For inhibitor experiments, 200nM of miRNA or AllStar inhibitor were used. Cells were incubated for 24 hours, after which cells were collected and total RNA extracted using the miRNeasy Mini kit (Qiagen). Two experiments, in triplicate, were performed for each mimic. Three experiments, in triplicate were performed for combined miR-323a-3p mimic and inhibition experiments. Reverse transcription and RTPCR were performed as described above, with GAPDH as the endogenous control.

\section{Luciferase assays:}

We examined two regions of ERBB4, corresponding to positions 6212-6685 (Region 1, R1) and 7219-7692 (Region 2, R2) of the 3'UTR of ERBB4 (uc002veg.1 of hg19), which contained the top predicted binding sites of miR-323a-3p by RNAHybrid, and Targetscan, respectively. Regions were ordered as gBlock gene fragments (IDT), and cloned between the HindIII and Spel sites of the pMIR-Report vector (Ambion) using T4 ligase (NEB). Constructs were verified by Sanger sequencing. Cells were transfected using HiPerfect with 1) 150ng of pMIR vector (R1, R2, or null), 2) 150ng of pRL-null vector, and 3) $20 \mathrm{nM}$ miR-323a-3p mimic or AllStar, or no treatment. After 24 hours, cells were collected and luciferase activity was assessed using the Dual Luciferase Assay (Promega) with a Spark 10M (Tecan). Untransfected cells were used to assess background luminescence, and values were subtracted from measured intensities of Firefly and Renilla luciferases. Values are reported as 
miR-323a and MDD

relative light units/second (RLU/s) and represent the activity of pMIR/pRL. Three experiments, in triplicate, were performed.

\section{Statistical analysis:}

For small RNA-seq data, fold changes $(\mathrm{FC})$ were calculated and t-tests were performed using log2-transformed normalized counts, in each brain region separately. Pearson correlations for miRNAs which displayed a $p$-value $<0.05$ and a FC of at least $20 \%$ were performed between transformed counts from each brain region in 33 subjects for which data was available for both brain regions, with a False Discovery Rate (FDR) cut-off of $5 \%$. One-tailed Pearson correlations between small RNA-seq and RT-PCR data were used for validation. Additionally, one-tailed Pearson correlations were used to characterize the relationship between miRNA expression and putative gene targets. RT-PCR of putative gene targets was non-normally distributed and was log2-transformed. Group differences were assessed using t-tests or Mann-Whitney tests, as appropriate. Twotailed Pearson correlations were used to assess the relationship between expression and linear variables (age, RIN, PMI). T-tests were used to examine the relationship between expression and dichotomous variables (gender, antidepressant history). For the animal behavioral data, $\mathrm{p}$-values were calculated using a two-tailed unpaired t-test. For cell culture experiments, we performed one-way ANOVAs, with Dunnett's or Tukey's posthoc tests for multiple comparisons. Normality of data was tested using Shapiro-Wilk or D'Agostino and Pearson tests. Equality of variances was tested using F-tests. All statistical analyses were conducted using SPSS V20 or GraphPad 5 and $p$-value $\leq 0.05$ was considered as significant, except where noted. 
miR-323a and MDD

Results:

\section{Small RNA-sequencing:}

We performed small RNA sequencing in 80 samples from the ACC (41 controls, 39 cases), and 37 samples from the habenula (13 controls, 24 cases) (Supplemental Table 1). Groups were matched for age, gender, and post-mortem interval (PMI) and pH. We obtained approximately 13.1 and 14.7 million reads per sample from the ACC, and habenula, respectively (Supplemental Table 2). After processing and alignment, each sample had an average of 375 or 353 miRNAs with at least 10 counts in the ACC and habenula, respectively.

As there is evidence for a relationship between the lateral habenula and the default-mode network, which includes the ACC, in depressive-like behaviors ${ }^{36}$, as well as for a direct connection and co-operative behavior between the ACC and the habenula ${ }^{37,38}$, we were interested in miRNAs whose expression was correlated between these two regions. We first identified miRNAs which displayed significantly correlated expression between the two brain regions. We identified 32 miRNAs which displayed significant positive correlations after correction for multiple testing (Supplemental Table 4). No miRNAs displayed negative correlations between these two regions. We next performed differential miRNA analyses between cases and controls, focusing on these 32 miRNAs. We identified four miRNAs, miR-204-5p, miR-320b, miR-323a-3p, and miR-331-3p, which displayed a fold change difference of greater than $20 \%$ in both regions, and whose expression differences were significant in at least one region (Figure 1). Expression differences were validated by quantitative RT-PCR (Supplemental Table 5).

\section{Targeted gene analysis:}

We used five target prediction algorithms to identify common putative targets of miR-204-5p, miR-320b, miR-323a-3p, and miR-331-3p. For each miRNA, we selected the genes that were predicted by all five algorithms, and identified 11 genes that were predicted targets of all four miRNAs (Figure 2A). Using RT-PCR, ten of these genes were found to be expressed in the ACC and habenula, and several were differentially expressed between cases and controls (Figure 2B and 2C). Furthermore, there were significant correlations between levels of the miRNAs and several of these genes (Supplemental Table 6).

\section{Analysis of miRNA-ERBB4 interactions in vitro:}

Among the predicted targets, erb-b2 receptor tyrosine kinase 4 (ERBB4), showed the greatest differential expression in the brain. Additionally, ERBB4 displayed the strongest correlations with levels of the differentially expressed miRNAs, such that it was negatively correlated with the expression of all four miRNAs in the habenula, as well as miR-320b in the ACC. To better characterize its relationship with the differentially expressed miRNAs, we performed in vitro experiments using miR-204-5p, miR-320b, miR-323a-3p, and miR331-3p mimics (Figure 3A). These experiments were performed in HEK293 cells, which endogenously express both ERBB4 and each of the miRNAs of interest (not shown). Among the miRNAs tested, only miR-323a-3p significantly altered the expression of ERBB4 relative to the negative control. We thus decided to focus on miR$323 a-3 p$ in subsequent experiments.

We further verified the relationship between miR-323a-3p and ERBB4 using a miR-323a-3p inhibitor. As shown in Figure $3 \mathrm{~B}$, treatment of cells with both the inhibitor and the mimic prevented the downregulation of ERBB4 observed by treatment with mimic alone.

In order to verify that miR-323a-3p directly targets the 3'UTR of ERBB4, we performed luciferase assays using two regions of the 3'UTR of ERBB4, which both contain predicted miR-323a-3p binding sites (Figure 3C). We found that miR-323a-3p significant decreased luciferase activity in the construct containing R1, with no effects on R2 or the empty vector. 


\section{Behavioral Effects of Overexpression and Knockdown of miR-323-3p in mouse ACC:}

To further explore the function of miR-323-3p in vivo, we generated adenoviruses (AAVs) that overexpress $(\mathrm{OE})$ or knock-down (KD) mouse miR-323-3p, which is equivalent to human miR-323a-3p (Supplemental Figure $1 A)$. These viruses were injected bilaterally in the mouse $\mathrm{Cg} 1 / 2$ (Figure $4 \mathrm{~B}$ and $\mathrm{C}$ ), a region that corresponds to the human ACC. We selected this region as it showed greater differential expression of miR-323a-3p in our human sample. In order to examine the effects of chronic miR-323-3p manipulation on anxiety- and/or depression-like behavior, the mice underwent various relevant behavioral tests (Figure 4D). In the open field test, miR-323-3p OE mice had a tendency to spend more time in the corners (Figure 4E), while inversely, inhibition of miR-323-3p resulted in mice spending less time in the corners compared to controls (Figure 4F). The elevated plus maze showed that OE mice spent more time in the centre of the maze, indicating that they peek their heads from the closed arms into the open arms. The miR-323-3p KD mice spent increased time in open arms of the elevated plus maze. The tail suspension was used to assess depressive-like behaviors: KD mice demonstrated increased struggling (mobility) in the tail suspension test. Results from the OE mice were not significantly different from their controls in these two tests. We generated an integrated emotionality score by combining data from the open field, elevated plus maze, and tail suspension tests (Figure 4J). Overall, our results indicate that increasing and decreasing miR-323-3p levels in the ACC yields increased and decreased emotionality, respectively.

\section{Relationship between miR-323-3p and Erbb4 in mice:}

In order to determine the relationship between miR-323-3p and Erbb4 expression in mice, we assessed Erbb4 levels in the ACC of the miR-323-3p OE and KD mice. As seen in Figure 4K, overexpression of miR-323-3p was associated with a trend for decreased expression of Erbb4, while miR-323-3p KD mice displayed significantly higher Erbb4 expression. These results agree well with our findings in humans. Moreover, comparison of the predicted miR-323 binding sites in the 3'UTR of ERBB4 in humans and mice indicates that the binding site in R1 is fully conserved, whereas there is a mismatch in the seed region of R2 (Figure 5). Although not conclusive, these results suggest that the effects of miR-323-3p on Erbb4 in mice may function through the R1 binding site. 


\section{Discussion:}

In this study, we identified four miRNAs which demonstrated elevated and correlated expression in the $A C C$ and habenula of depressed individuals. Additionally, we found that miR-323a-3p influences the expression of ERBB4, which displayed decreased expression in both brain regions. This relationship was also observed in mice in which the levels of miR-323-3p had been knocked down and overexpressed. Finally, manipulation of miR-323-3p expression in the ACC induced behavioral effects in mice.

MiR-323a-3p demonstrates elevated expression in the brain, where it demonstrates particularly enriched expression in synaptoneurosomes in the cortex and hippocampus ${ }^{39}$. Although it has not been well-studied in psychiatry, it has been shown to be upregulated in the brains of rats exposed to prenatal stress ${ }^{40}$, and plasma levels appear to be related to mild cognitive impairment in humans ${ }^{41,42}$. In our study, we found that overexpression of mouse miR-323-3p in the brain was associated with increased anxiety-like behaviors, whereas decreasing miR-323-3p levels reduced anxiety- and depressive-like behaviors. These findings agree well with our results in depressed individuals, and add support for a causal role of elevated levels of this miRNA in psychopathology.

A number of mRNA targets of miR-323a have been identified, and include insulin-like growth factor 1 (IGF1) ${ }^{43}$, tumor protein p73 (TP73) ${ }^{44}$, brain protein I3 (BRI3) ${ }^{45}$, and fragile X mental retardation 1 (FMR1) ${ }^{46}$. MiR-323a is located in a large cluster of 54 miRNAs, found within the imprinted DLK1-DIO3 locus on 14q32 (reviewed in ${ }^{47}$ ). The miRNAs within this cluster are maternally expressed, and their imprinting and expression is epigenetically regulated through DNA methylation at several genomic regions within this locus. In our sample, we found a strong correlation between many of the miRNAs in this cluster (Supplemental Figure 2), including miR-323a. A number of studies have demonstrated that altered functioning of this locus influences neurogenesis ${ }^{48}$ and pleuripotency ${ }^{49}$. Interestingly, a recent study investigating glioblastoma found decreased levels of the expression of 13 miRNAs within this cluster, including miR-323a, and highlighted neuregulin signaling as the most enriched pathway among putative target genes ${ }^{50}$. More recently, several miRNAs in this cluster, including miR-323a, were found to be implicated in Parkinson's Disease, and may possess neuroprotective properties ${ }^{51}$. Additionally, ErbB signaling was among the most enriched pathways of predicted miRNA targets. Interestingly, these miRNAs also contained binding sites for the transcription factor cAMPresponsive element-binding protein 1 (CREB1), which has been previously implicated in major depressive disorder and antidepressant responses ${ }^{52}$. This is particularly interesting given that in our study, we observed a trend for elevated miR-323a expression in the ACC, in subjects with a positive 3-month history of antidepressant use compared to untreated individuals with MDD and healthy controls $(p=0.053$, one-way ANOVA). It is unclear whether upregulation of miR-323a is related to the antidepressant treatment itself, or if this miRNA is particularly enriched in individuals who are unresponsive to treatment.

In the present study, we found that miR-323a-3p influences the expression of ERBB4. This tyrosine-protein kinase receptor, which displays highly enriched expression in the brain (Supplemental Figure 3A-C), is part of the epidermal growth factor receptor subfamily, and is known to bind to several neuregulins and related growth factors. Dysregulated functioning of this gene has been implicated in schizophrenia and mood disorders 53-55, as well as both anxiety- and depressive-like behaviors in rodents ${ }^{56,57}$. Moreover, it appears to be involved in the antidepressant effects of ketamine ${ }^{58}$. Here, we observed downregulated expression of ERBB4 in both the ACC and the habenula. Furthermore, we found that at least in the mouse brain, both miR-323a (Supplemental Figure 3D) and ERBB4 are expressed within multiple brain regions. This opens up the possibility that the regulation of ERBB4 by miR-323a is not exclusively restricted to the ACC region and can be generalized to potentially multiple brain regions, adding further support for an important functional role of this gene in psychiatric disorders.

In addition to miR-323a-3p, we also identified overexpression of miR-204-5p, miR-320b, and miR-331-3p in the brains of depressed individuals. MiR-204 appears to have numerous important functions in the nervous system, including axon regeneration ${ }^{59}$ and hippocampal ageing ${ }^{60}$, stem cell differentiation ${ }^{61}$, and notable targets include EPH receptor B2 (EPHB2) ${ }^{60}$, tropomyosin related kinase type B (TrkB) ${ }^{62}$, and ERBB3 ${ }^{63}$. Additionally, this miRNA has been associated with schizophrenia ${ }^{64}$, and is responsive to social enrichment in a rat model of fetal alcohol disorder ${ }^{65}$. Similar to miR-323a, miR-204 displays enriched expression in the brain compared to other tissues ${ }^{7}$. MiR-320b has also been associated with schizophrenia ${ }^{66}$ and brain development 
miR-323a and MDD

${ }^{67}$, and some of its mRNA targets include cyclin dependent kinase 6 (CDK6) ${ }^{68}$ and distal-less homeobox 5 (DLX5) ${ }^{69}$. Finally, miR-331 has been implicated in epilepsy ${ }^{70}$ and the protective effects of valproic acid treatment following ischemia ${ }^{71}$, and has been shown to target ERBB2 ${ }^{72}$, neuropilin-2 (NRP-2) ${ }^{73}$ and astrocyte-elevated gene-1 (AEG-1) ${ }^{74}$.

As mentioned above, there have been relatively few high- throughput miRNA studies performed in brain tissue of individuals with MDD. Although four studies focused on the prefrontal cortex ${ }^{2,12-14}$, they have failed to identify consistent alterations, which may be due to differences in pre-frontal cortical areas being examined, technologies used to quantify miRNAs, or statistical methods for multiple testing. Indeed, as exemplified by miRNAs in the $14 \mathrm{q} 32$ cluster, many miRNAs display co-regulation and coordinated expression, such that correction methods assuming independence between miRNAs are inappropriate. Further, differences in RNA extraction, miRNA quantification, and normalization methods can greatly impact which miRNAs are detectable ${ }^{75}$, as well as their relative abundances in a sample, making comparison across studies to be challenging. One smaller-scale study investigated 29 miRNAs in depression and bipolar disorder in the ACC, however they did not examine the miRNAs we identified as significant in our sample ${ }^{76}$. To date, no studies have examined the expression of miRNAs in the human habenula. However, one study which examined miRNAs in a learned helplessness model in rats found ErbB signaling to be one of the significantly targeted pathways of affected miRNAs ${ }^{77}$, strengthening support for the importance of this pathway in the habenula.

Finally, it must be noted that our human studies were performed in individuals with MDD who died by suicide. As such, we are unable to differentiate between the effects associated with MDD from those which may be attributable to suicide. However, we observed similar changes to those observed in humans using a mouse model of depressive-like behaviors, suggesting that at least some of these alterations are more likely to reflect processes related to depression. Future studies will be needed in order to better disentangle these effects.

In conclusion, we identified several miRNAs which displayed elevated levels in the brains of individuals with depression. Furthermore, we found that miR-323-3p is associated with anxiety- and depressive-like behaviors in mice, and influences the expression of several genes, most notably ERBB4. Future studies should better characterize the role of miR-323a-3p and ERBB4 in depression and related behaviors. 
G.T. holds a Canada Research Chair (Tier 1 ) and is supported by grants from the Canadian Institute of Health Research (CIHR) (FDN148374, FRN 141899, ENP161427), and by the Fonds de recherche du Québec - Santé (FRQS) (EGM141899) through the Quebec Network on Suicide, Mood Disorders and Related Disorders. This project was jointly funded by FRQS (EGM141899), the Canadian Institutes of Health Research (FRN 141899), the German Bundesministerium für Bildung and Forschung (01KU1504) and the French Agence Nationale de la Recherche (ANR-15-EPIG-0003-03 and ANR-15-EPIG-0003-04). Additionally, this research was undertaken thanks in part to funding from the Canada First Research Excellence Fund, awarded to McGill University for the Healthy Brains for Healthy Lives (HBHL) initiative (4b-CF-5). A.C. is the incumbent of the Vera and John Schwartz Family Professorial Chair at the Weizmann Institute and is the Head of the Max Planck SocietyWeizmann Institute of Science Laboratory for Experimental Neuropsychiatry and Behavioral Neurogenetics. This project was funded by the Federal Ministry of Education and Research under the funding code 01KU1501A (A.C.) and supported by a research grant from the Israel Science Foundation (1565/15, A.C.). We thank A. Varga and all the caretakers of the Max Planck Institute of Psychiatry for their devoted assistance with animal care.

\section{Conflict of Interest:}

The authors declare no conflict of interest.

\section{Supplementary Information:}

Supplementary information is available at the Molecular Psychiatry website. 


\section{References}

1. Disease GBD, Injury I, Prevalence C. Global, regional, and national incidence, prevalence, and years lived with disability for 354 diseases and injuries for 195 countries and territories, 1990-2017: a systematic analysis for the Global Burden of Disease Study 2017. Lancet 2018; 392(10159): 1789-1858.

2. Lopez JP, Lim R, Cruceanu C, Crapper L, Fasano C, Labonte B et al. miR-1202 is a primate-specific and brain-enriched microRNA involved in major depression and antidepressant treatment. Nat Med 2014; 20(7): 764-768.

3. Fries GR, Carvalho AF, Quevedo J. The miRNome of bipolar disorder. J Affect Disord 2018; 233: 110-116.

4. Liu Y, Chang X, Hahn CG, Gur RE, Sleiman PAM, Hakonarson H. Non-coding RNA dysregulation in the amygdala region of schizophrenia patients contributes to the pathogenesis of the disease. Transl Psychiatry 2018; 8(1): 44.

5. Bartel DP. Metazoan MicroRNAs. Cell 2018; 173(1): 20-51.

6. Issler O, Chen A. Determining the role of microRNAs in psychiatric disorders. Nat Rev Neurosci 2015; 16(4): 201-212.

7. Ludwig N, Leidinger $\mathrm{P}$, Becker $\mathrm{K}$, Backes $\mathrm{C}$, Fehlmann $\mathrm{T}$, Pallasch $\mathrm{C}$ et al. Distribution of miRNA expression across human tissues. Nucleic Acids Res 2016; 44(8): 3865-3877.

8. Pons-Espinal M, de Luca E, Marzi MJ, Beckervordersandforth R, Armirotti A, Nicassio F et al. Synergic Functions of miRNAs Determine Neuronal Fate of Adult Neural Stem Cells. Stem Cell Reports 2017; 8(4): 1046-1061.

9. Shin J, Shin Y, Oh SM, Yang H, Yu WJ, Lee JP et al. MiR-29b controls fetal mouse neurogenesis by regulating ICAT-mediated Wnt/beta-catenin signaling. Cell Death Dis 2014; 5: e1473.

10. Wei CW, Luo T, Zou SS, Wu AS. Research progress on the roles of microRNAs in governing synaptic plasticity, learning and memory. Life Sci 2017; 188: 118-122.

11. Dwivedi Y. MicroRNAs in depression and suicide: Recent insights and future perspectives. J Affect Disord 2018; 240: 146-154.

12. Smalheiser NR, Lugli G, Rizavi HS, Torvik VI, Turecki G, Dwivedi Y. MicroRNA expression is downregulated and reorganized in prefrontal cortex of depressed suicide subjects. PLoS One 2012; 7(3): e33201.

13. Smalheiser NR, Lugli G, Zhang H, Rizavi H, Cook EH, Dwivedi Y. Expression of microRNAs and other small RNAs in prefrontal cortex in schizophrenia, bipolar disorder and depressed subjects. PLoS One 2014; 9(1): e86469.

14. Pantazatos SP, Huang YY, Rosoklija GB, Dwork AJ, Arango V, Mann JJ. Whole-transcriptome brain expression and exon-usage profiling in major depression and suicide: evidence for altered glial, endothelial and ATPase activity. Mol Psychiatry 2017; 22(5): 760-773. 
miR-323a and MDD

15. Roy B, Wang Q, Palkovits M, Faludi G, Dwivedi Y. Altered miRNA expression network in locus coeruleus of depressed suicide subjects. Sci Rep 2017; 7(1): 4387.

16. Forero DA, Guio-Vega GP, Gonzalez-Giraldo Y. A comprehensive regional analysis of genome-wide expression profiles for major depressive disorder. J Affect Disord 2017; 218: 86-92.

17. Sambataro F, Doerig N, Hanggi J, Wolf RC, Brakowski J, Holtforth MG et al. Anterior cingulate volume predicts response to psychotherapy and functional connectivity with the inferior parietal cortex in major depressive disorder. Eur Neuropsychopharmacol 2018; 28(1): 138-148.

18. Liu J, Xu X, Luo $Q$, Luo $Y$, Chen $Y$, Lui $S$ et al. Brain grey matter volume alterations associated with antidepressant response in major depressive disorder. Sci Rep 2017; 7(1): 10464.

19. Dandekar MP, Fenoy AJ, Carvalho AF, Soares JC, Quevedo J. Deep brain stimulation for treatmentresistant depression: an integrative review of preclinical and clinical findings and translational implications. Mol Psychiatry 2018; 23(5): 1094-1112.

20. Browne CA, Hammack R, Lucki I. Dysregulation of the Lateral Habenula in Major Depressive Disorder. Front Synaptic Neurosci 2018; 10: 46.

21. Huang L, Xi Y, Peng Y, Yang Y, Huang X, Fu Y et al. A Visual Circuit Related to Habenula Underlies the Antidepressive Effects of Light Therapy. Neuron 2019.

22. Gosnell SN, Curtis KN, Velasquez K, Fowler JC, Madan A, Goodman W et al. Habenular connectivity may predict treatment response in depressed psychiatric inpatients. J Affect Disord 2019; 242: 211-219.

23. Dumais $A$, Lesage $A D$, Alda $M$, Rouleau $G$, Dumont $M$, Chawky $N$ et al. Risk factors for suicide completion in major depression: a case-control study of impulsive and aggressive behaviors in men. Am J Psychiatry 2005; 162(11): 2116-2124.

24. Song L, Florea L, Langmead B. Lighter: fast and memory-efficient sequencing error correction without counting. Genome Biol 2014; 15(11): 509.

25. Chen CJ, Servant N, Toedling J, Sarazin A, Marchais A, Duvernois-Berthet E et al. ncPRO-seq: a tool for annotation and profiling of ncRNAs in sRNA-seq data. Bioinformatics 2012; 28(23): 3147-3149.

26. Kozomara A, Griffiths-Jones S. miRBase: annotating high confidence microRNAs using deep sequencing data. Nucleic Acids Res 2014; 42(Database issue): D68-73.

27. Love MI, Huber $\mathrm{W}$, Anders S. Moderated estimation of fold change and dispersion for RNA-seq data with DESeq2. Genome Biol 2014; 15(12): 550.

28. Dweep H, Gretz N. miRWalk2.0: a comprehensive atlas of microRNA-target interactions. Nat Methods 2015; 12(8): 697.

29. Betel D, Koppal A, Agius P, Sander C, Leslie C. Comprehensive modeling of microRNA targets predicts functional non-conserved and non-canonical sites. Genome Biol 2010; 11(8): R90.

30. Miranda KC, Huynh T, Tay Y, Ang YS, Tam WL, Thomson AM et al. A pattern-based method for the identification of MicroRNA binding sites and their corresponding heteroduplexes. Cell 2006; 126(6): 1203-1217. 
miR-323a and MDD

31. Rehmsmeier $M$, Steffen $P$, Hochsmann $M$, Giegerich $R$. Fast and effective prediction of microRNA/target duplexes. RNA 2004; 10(10): 1507-1517.

32. Lewis BP, Burge $\mathrm{CB}$, Bartel DP. Conserved seed pairing, often flanked by adenosines, indicates that thousands of human genes are microRNA targets. Cell 2005; 120(1): 15-20.

33. Schroeder M, Drori Y, Ben-Efraim YJ, Chen A. Hypothalamic miR-219 regulates individual metabolic differences in response to diet-induced weight cycling. Mol Metab 2018; 9: 176-186.

34. Ebert MS, Neilson JR, Sharp PA. MicroRNA sponges: competitive inhibitors of small RNAs in mammalian cells. Nat Methods 2007; 4(9): 721-726.

35. Guilloux JP, Seney M, Edgar N, Sibille E. Integrated behavioral z-scoring increases the sensitivity and reliability of behavioral phenotyping in mice: relevance to emotionality and sex. $J$ Neurosci Methods 2011; 197(1): 21-31.

36. Clemm von Hohenberg C, Weber-Fahr W, Lebhardt P, Ravi N, Braun U, Gass N et al. Lateral habenula perturbation reduces default-mode network connectivity in a rat model of depression. Transl Psychiatry 2018; 8(1): 68.

37. Kawai T, Yamada H, Sato N, Takada M, Matsumoto M. Roles of the Lateral Habenula and Anterior Cingulate Cortex in Negative Outcome Monitoring and Behavioral Adjustment in Nonhuman Primates. Neuron 2015; 88(4): 792-804.

38. Chiba T, Kayahara T, Nakano K. Efferent projections of infralimbic and prelimbic areas of the medial prefrontal cortex in the Japanese monkey, Macaca fuscata. Brain Res 2001; 888(1): 83-101.

39. Pichardo-Casas I, Goff LA, Swerdel MR, Athie A, Davila J, Ramos-Brossier M et al. Expression profiling of synaptic microRNAs from the adult rat brain identifies regional differences and seizure-induced dynamic modulation. Brain Res 2012; 1436: 20-33.

40. Zucchi FC, Yao Y, Ward ID, Ilnytskyy Y, Olson DM, Benzies K et al. Maternal stress induces epigenetic signatures of psychiatric and neurological diseases in the offspring. PLoS One 2013; 8(2): e56967.

41. Sheinerman KS, Tsivinsky VG, Crawford F, Mullan MJ, Abdullah L, Umansky SR. Plasma microRNA biomarkers for detection of mild cognitive impairment. Aging (Albany NY) 2012; 4(9): 590-605.

42. Sheinerman KS, Tsivinsky VG, Abdullah L, Crawford F, Umansky SR. Plasma microRNA biomarkers for detection of mild cognitive impairment: biomarker validation study. Aging (Albany NY) 2013; 5(12): 925-938.

43. Wang $T$, Liu $Y$, Lv $M$, Xing $Q$, Zhang $Z$, He $X$ et al. miR-323-3p regulates the steroidogenesis and cell apoptosis in polycystic ovary syndrome (PCOS) by targeting IGF-1. Gene 2019; 683: 87-100.

44. Gao $Q$, Zheng J. microRNA-323 upregulation promotes prostate cancer growth and docetaxel resistance by repressing p73. Biomed Pharmacother 2018; 97: 528-534.

45. Yang L, Xiong Y, Hu XF, Du YH. MicroRNA-323 regulates ischemia/reperfusion injury-induced neuronal cell death by targeting BRI3. Int J Clin Exp Pathol 2015; 8(9): 10725-10733.

46. Yi YH, Sun XS, Qin JM, Zhao QH, Liao WP, Long YS. Experimental identification of microRNA targets on the 3' untranslated region of human FMR1 gene. J Neurosci Methods 2010; 190(1): 34-38. 
47. Benetatos L, Hatzimichael E, Londin E, Vartholomatos $\mathrm{G}$, Loher P, Rigoutsos I et al. The microRNAs within the DLK1-DIO3 genomic region: involvement in disease pathogenesis. Cell Mol Life Sci 2013; 70(5): 795-814.

48. Ferron SR, Charalambous M, Radford E, McEwen K, Wildner H, Hind E et al. Postnatal loss of Dlk1 imprinting in stem cells and niche astrocytes regulates neurogenesis. Nature 2011; 475(7356): 381-385.

49. Stadtfeld $M$, Apostolou $E$, Akutsu $H$, Fukuda A, Follett $P$, Natesan $S$ et al. Aberrant silencing of imprinted genes on chromosome 12qF1 in mouse induced pluripotent stem cells. Nature 2010; 465(7295): 175181.

50. Shahar T, Granit A, Zrihan D, Canello T, Charbit H, Einstein O et al. Expression level of miRNAs on chromosome 14q32.31 region correlates with tumor aggressiveness and survival of glioblastoma patients. J Neurooncol 2016; 130(3): 413-422.

51. Ravanidis S, Bougea A, Papagiannakis N, Koros C, Simitsi AM, Pachi I et al. Validation of differentially expressed brain-enriched microRNAs in the plasma of PD patients. Ann Clin Transl Neurol 2020.

52. Niciu MJ, lonescu DF, Mathews DC, Richards EM, Zarate CA, Jr. Second messenger/signal transduction pathways in major mood disorders: moving from membrane to mechanism of action, part I: major depressive disorder. CNS Spectr 2013; 18(5): 231-241.

53. Goes FS, Rongione M, Chen YC, Karchin R, Elhaik E, Bipolar Genome S et al. Exonic DNA sequencing of ERBB4 in bipolar disorder. PLoS One 2011; 6(5): e20242.

54. Chung DW, Chung Y, Bazmi HH, Lewis DA. Altered ErbB4 splicing and cortical parvalbumin interneuron dysfunction in schizophrenia and mood disorders. Neuropsychopharmacology 2018; 43(12): 2478-2486.

55. Chong VZ, Thompson M, Beltaifa S, Webster MJ, Law AJ, Weickert CS. Elevated neuregulin-1 and ErbB4 protein in the prefrontal cortex of schizophrenic patients. Schizophr Res 2008; 100(1-3): 270-280.

56. Bi LL, Sun XD, Zhang J, Lu YS, Chen YH, Wang J et al. Amygdala NRG1-ErbB4 is critical for the modulation of anxiety-like behaviors. Neuropsychopharmacology 2015; 40(4): 974-986.

57. Dang R, Cai H, Zhang L, Liang D, Lv C, Guo Y et al. Dysregulation of Neuregulin-1/ErbB signaling in the prefrontal cortex and hippocampus of rats exposed to chronic unpredictable mild stress. Physiol Behav 2016; 154: 145-150.

58. Wang N, Zhang GF, Liu XY, Sun HL, Wang XM, Qiu LL et al. Downregulation of neuregulin 1-ErbB4 signaling in parvalbumin interneurons in the rat brain may contribute to the antidepressant properties of ketamine. J Mol Neurosci 2014; 54(2): 211-218.

59. Su LN, Song XQ, Xue ZX, Zheng CQ, Yin HF, Wei HP. Network analysis of microRNAs, transcription factors, and target genes involved in axon regeneration. J Zhejiang Univ Sci B 2018; 19(4): 293-304.

60. Mohammed CP, Rhee H, Phee BK, Kim K, Kim HJ, Lee H et al. miR-204 downregulates EphB2 in aging mouse hippocampal neurons. Aging Cell 2016; 15(2): 380-388.

61. Lepko T, Pusch $M$, Muller T, Schulte D, Ehses J, Kiebler $M$ et al. Choroid plexus-derived miR-204 regulates the number of quiescent neural stem cells in the adult brain. EMBO J 2019; 38(17): e100481. 
miR-323a and MDD

62. Xiang L, Ren Y, Li X, Zhao W, Song Y. MicroRNA-204 suppresses epileptiform discharges through regulating TrkB-ERK1/2-CREB signaling in cultured hippocampal neurons. Brain Res 2016; 1639: 99-107.

63. Shi Y, Chen X, Xi B, Yu X, Ouyang J, Han C et al. SNP rs3202538 in 3'UTR region of ErbB3 regulated by miR-204 and miR-211 promote gastric cancer development in Chinese population. Cancer Cell Int 2017; 17: 81.

64. Cammaerts S, Strazisar M, Smets B, Weckhuysen S, Nordin A, De Jonghe P et al. SchizophreniaAssociated MIR204 Regulates Noncoding RNAs and Affects Neurotransmitter and lon Channel Gene Sets. PLoS One 2015; 10(12): e0144428.

65. Ignacio C, Mooney SM, Middleton FA. Effects of Acute Prenatal Exposure to Ethanol on microRNA Expression are Ameliorated by Social Enrichment. Front Pediatr 2014; 2: 103.

66. Wang $\mathrm{Y}$, Wang J, Guo T, Peng Y, Wang K, Bai K et al. Screening of schizophrenia associated miRNAs and the regulation of miR-320a-3p on integrin beta1. Medicine (Baltimore) 2019; 98(8): e14332.

67. Somel M, Liu X, Tang L, Yan Z, Hu H, Guo S et al. MicroRNA-driven developmental remodeling in the brain distinguishes humans from other primates. PLoS Biol 2011; 9(12): e1001214.

68. Tadano T, Kakuta Y, Hamada S, Shimodaira Y, Kuroha M, Kawakami Y et al. MicroRNA-320 family is downregulated in colorectal adenoma and affects tumor proliferation by targeting CDK6. World $J$ Gastrointest Oncol 2016; 8(7): 532-542.

69. Laxman N, Mallmin H, Nilsson O, Kindmark A. miR-203 and miR-320 Regulate Bone Morphogenetic Protein-2-Induced Osteoblast Differentiation by Targeting Distal-Less Homeobox 5 (Dlx5). Genes (Basel) 2016; 8(1).

70. Kretschmann A, Danis B, Andonovic L, Abnaof K, van Rikxoort M, Siegel $F$ et al. Different microRNA profiles in chronic epilepsy versus acute seizure mouse models. J Mol Neurosci 2015; 55(2): 466-479.

71. Hunsberger JG, Fessler EB, Wang Z, Elkahloun AG, Chuang DM. Post-insult valproic acid-regulated microRNAs: potential targets for cerebral ischemia. Am J Transl Res 2012; 4(3): 316-332.

72. Epis MR, Giles KM, Barker A, Kendrick TS, Leedman PJ. miR-331-3p regulates ERBB-2 expression and androgen receptor signaling in prostate cancer. J Biol Chem 2009; 284(37): 24696-24704.

73. Epis MR, Giles KM, Candy PA, Webster RJ, Leedman PJ. miR-331-3p regulates expression of neuropilin-2 in glioblastoma. J Neurooncol 2014; 116(1): 67-75.

74. Chen L, Ma G, Cao X, An X, Liu X. MicroRNA-331 inhibits proliferation and invasion of melanoma cells by targeting astrocyte-elevated gene-1. Oncol Res 2018.

75. Lopez JP, Diallo A, Cruceanu C, Fiori LM, Laboissiere S, Guillet I et al. Biomarker discovery: quantification of microRNAs and other small non-coding RNAs using next generation sequencing. $B M C$ Med Genomics 2015; 8: 35.

76. Azevedo JA, Carter BS, Meng F, Turner DL, Dai M, Schatzberg AF et al. The microRNA network is altered in anterior cingulate cortex of patients with unipolar and bipolar depression. J Psychiatr Res 2016; 82: 58-67. 
miR-323a and MDD

77. Svenningsen K, Veno MT, Henningsen K, Mallien AS, Jensen L, Christensen T et al. MicroRNA Profiling in the Medial and Lateral Habenula of Rats Exposed to the Learned Helplessness Paradigm: Candidate Biomarkers for Susceptibility and Resilience to Inescapable Shock. PLoS One 2016; 11(8): e0160318.

78. Paxinos G, Franklin KBJ, Franklin KBJ. The mouse brain in stereotaxic coordinates. 2nd edn. Academic Press: San Diego, 2001. 
Figure 1: miRNAs displaying differential expression in both brain regions. Normalized log2 transformed counts are shown for ACC (grey) and habenula (blue) for (A) miR-204-5p, ACC ( $p=0.01$, fold change $(F C)=1.25)$, (B) miR-320b, ACC ( $p=0.19, \mathrm{FC}=1.23)$, (C) miR-323a-3p, ACC ( $p=0.04, \mathrm{FC}=1.25)$, (D) miR-331-3p, ACC ( $p=0.01$, $\mathrm{FC}=1.36)$, (E) miR-204-5p, habenula $(p=0.14, F C=1.30)$, (F) miR-320b, habenula $(p=0.03, F C=1.46),(G)$ miR323a-3p, habenula ( $p=0.06, F C=1.30$, and $(H)$ miR-331-3p, habenula $(p=0.03, F C=1.22)$. $F C$ represent changes in cases (ACC: 39 , habenula: 24 ) relative to healthy controls (ACC: 41, habenula: 13 ). Note that counts from the ACC and habenula were normalized separately. MiRNA expression was normally distributed and group differences were assessed using t-tests.

Figure 2: Overlapping gene targets of miR-204-5p, miR-320b, miR-323a-3p, and miR-331-3p. (A) Overlap of predicted targets of miR-204, miR-320b, miR-323a, and miR-331. (B) Gene expression of overlapping genes in the ACC was assessed by RT-PCR. (C) Gene expression assessed by RT-PCR in the habenula. Note that TP73, which was also a predicted target, was not expressed in our sample. Group differences were assessed using ttests or Mann-Whitney tests, as appropriate. Error bars represent standard error of the mean.

Figure 3: Effects of miRNA treatment on ERBB4 expression in HEK293 cells. (A) Cells were either treated for 24 hours with 20nM miRNA mimics, 20nM AllStar negative control (NT), or untreated (U). Expression (and SD) was quantified using GAPDH as the endogenous control. Mir-323a-3p significantly decreased ERBB4 expression relative to the negative control ( $p=0.049$, one-way ANOVA, Dunnetts multiple comparison). (B) Cells were treated with $20 \mathrm{nM}$ mimic $(\mathrm{m})$ and/or 200nM inhibitor (i), directed against miR-323a, or left untreated (U). Expression (and SD) was quantified using GAPDH as the endogenous control. Treatment with mimic and inhibitor $(\mathrm{m}+\mathrm{i})$ did not alter expression relative to untreated cells $(p>0.99$, one-way ANOVA, Dunnetts multiple comparison). (C) Cells were treated for 24 hours with 150ng of pMIR vector (R1, R2, or pMIR), 2) 150ng of pRLnull vector, and 3) $20 \mathrm{nM}$ miR-323a-3p mimic $(\mathrm{m})$ or AllStar (NT). Firefly (pMIR) luciferase activity was normalized to renilla luciferase ( $\mathrm{pRL}$-null). Treatment with mimic decreased the expression of R1 relative to NT and $\mathrm{pMIR}$ treated with mimic ( $p=0.007, p<0.0001$, one-way ANOVA, Tukey's multiple comparison). No changes were observed for R2 or pMIR when treated with mimic compared to NT, or no treatment (not shown).

Figure 4: Manipulation of miR-323-3p in the mouse ACC modulates anxiety- and depression-like behaviors. (A) Schematic overview of EF1a promotor driven miR-323 overexpression (in blue) and miR-323 inhibiting sponge (in red) constructs with their respective controls. (B) Coronal map with stereotactic coordinates of the region targeted for viral manipulation. Image was adapted from the mouse brain atlas ${ }^{78}$ (C) Representative images of mouse brains injected in the ACC (Cg region 1 and 2) with miR-scrambled, miR-323 overexpression, miR-control-sponge and miR-323-sponge AAV virus respectively. The grayscale images were color inverted to show the fluorescent GFP signal expressed by the viral constructs as black signal highlighting the site where each of the viruses are expressed. (D) The order of the behavioral tests that were run and a figure legend for all subsequent graphs. (E and $\mathbf{F}$ ) The OF test showing relative time spent in the centre, corner and total distance traveled in meters. (D) Mice injected with miR-323 overexpression virus have a tendency to spend more time in the corners $(p=0.0821)$. (E) miR-323 sponge injected mice spent significantly less time in the corners $(p=0.0248)$. ( $\mathbf{E}$ and $\mathbf{F})$ No difference was observed in total distance traveled. ( $\mathbf{G}$ and $\mathbf{H})$ The EPM test with relative time spent in the open arms, centre and the closed arms of the maze. (F) MiR-323 overexpression mice spent more time in the center of the maze compared to miR-scrambled injected mice $(p=0.0142),(\mathbf{G})$ while miR-323-sponge mice displayed a relative increase in time spent in the open arms compared to control mice $(p=0.029)$. (I) The TST showing the relative mobility time. Inhibition of miR-323 significantly increases mobility time compared to control sponge injected mice $(p=0.0369)$. (J) Emotionality z-score integrating the OF, EPM and TST. MiR-323 overexpression mice have a tendency to have a higher emotionality score compared to controls $(p=0.0548)$. Inhibiting miR-323 decreases the emotionality score compared to control mice $(p=0.0115)$. Groups consisted of $n=9$ for miR-323, miR-scramble, miR-323-sponge and $n=10$ for miR-control-sponge. (K) Mice overexpressing miR-323 in the ACC displayed a trend for decreased expression of Erbb4 ( $p=0.09)$. Mice treated with a miR-323 sponge displayed significantly elevated expression of Erbb4 $(p=0.006)$. 
miR-323a and MDD

Figure 5: Comparison of Human and Mouse miR-323 binding sites in the 3'UTR of ERBB4. Region 1, which was regulated by miR-323a-3p in vitro is fully conserved between human and mouse, whereas a mismatch between species is found in the predicted seed region of Region 2. 
A

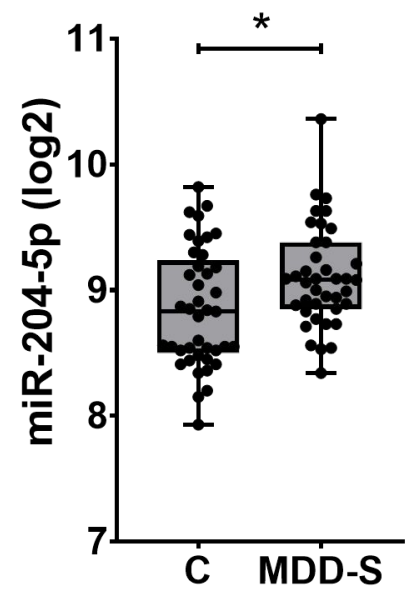

E

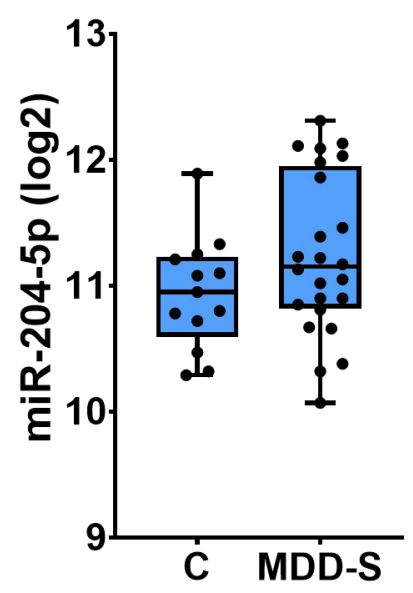

B

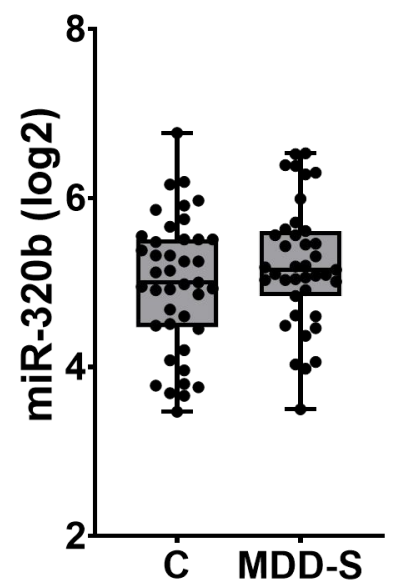

F

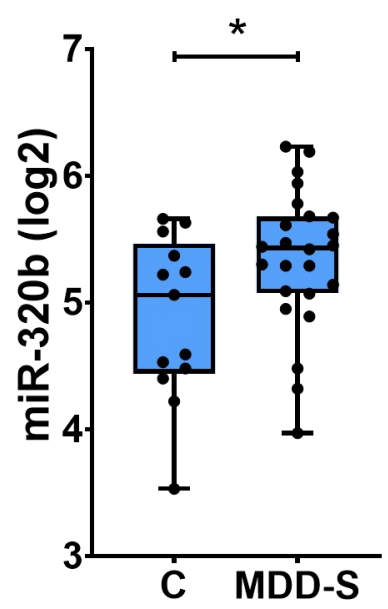

C

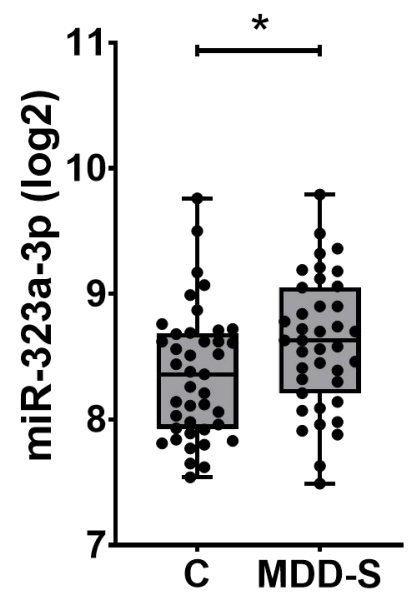

G

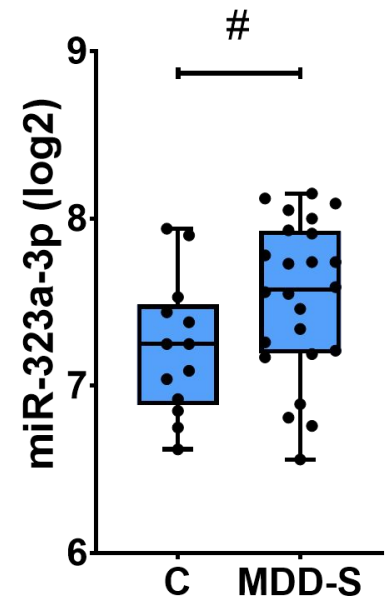

D

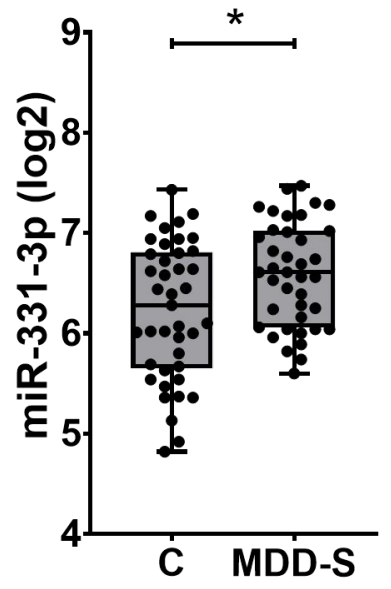

H

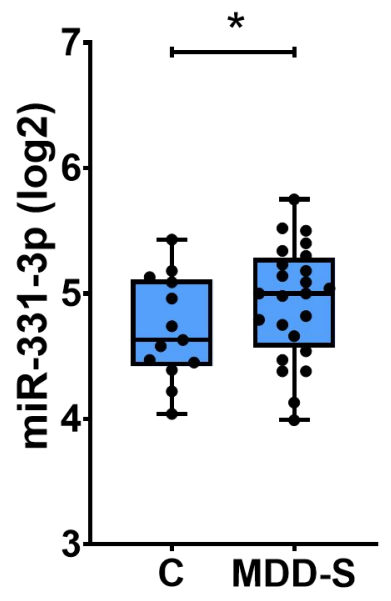


A
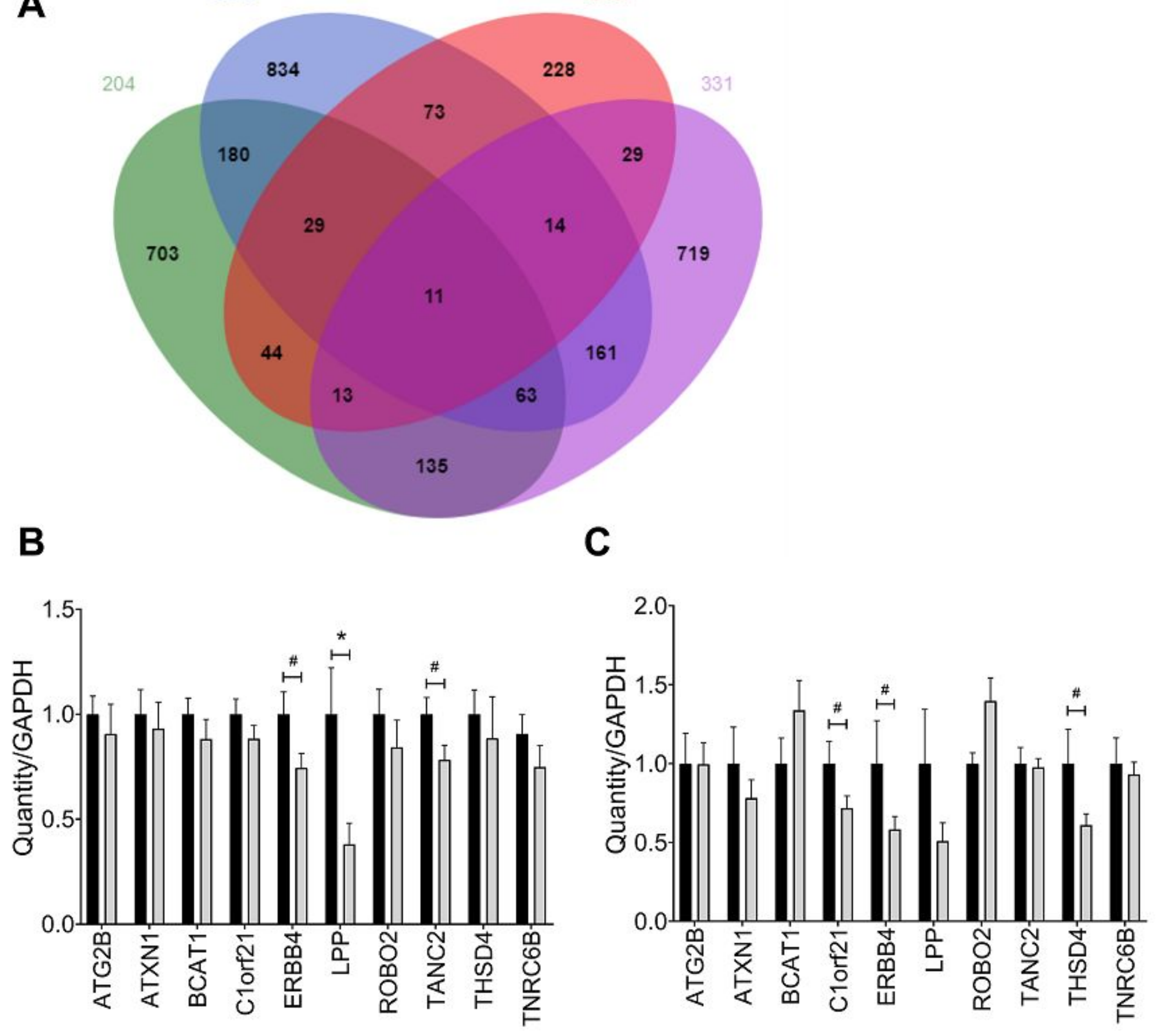
A

B
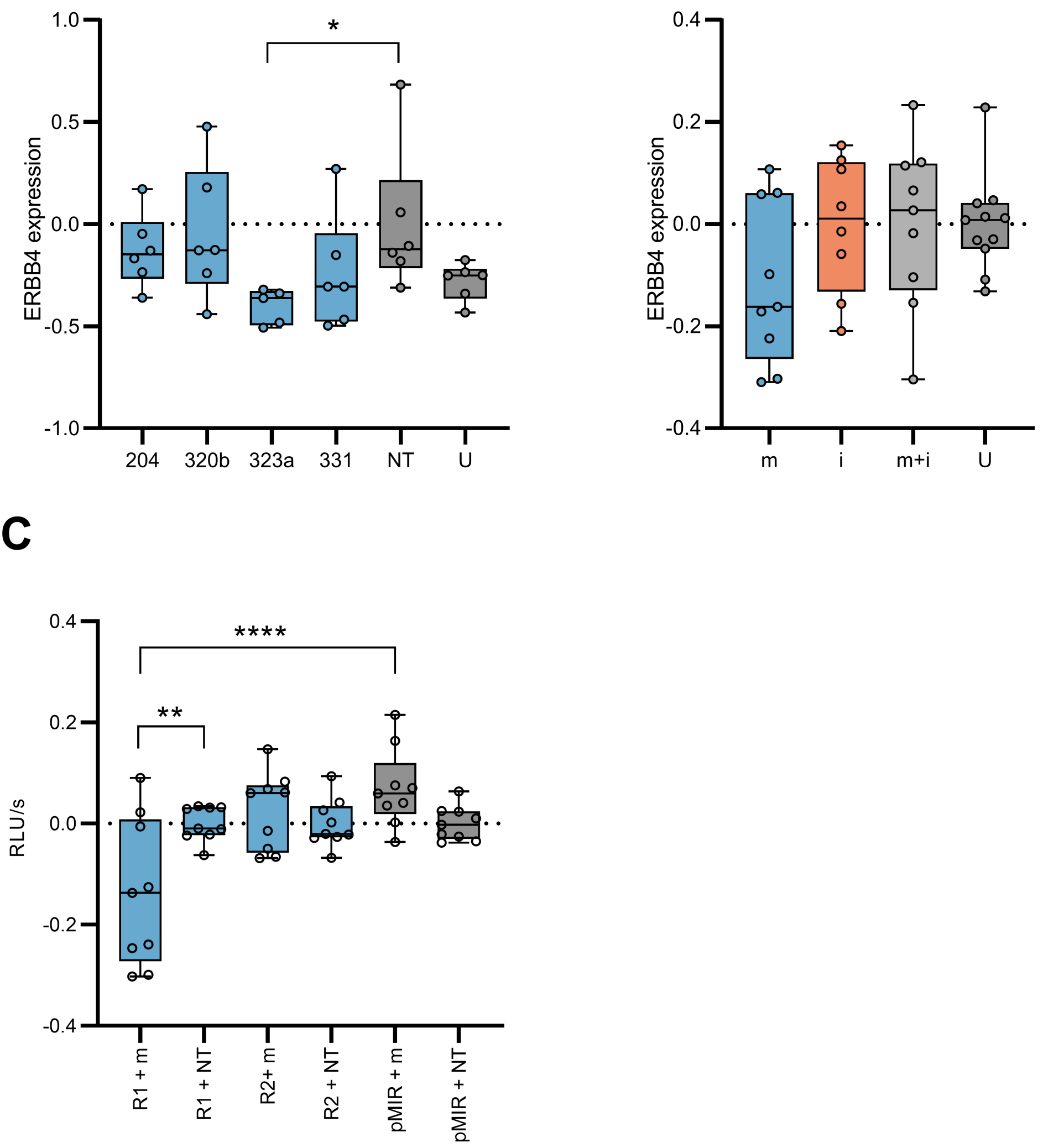


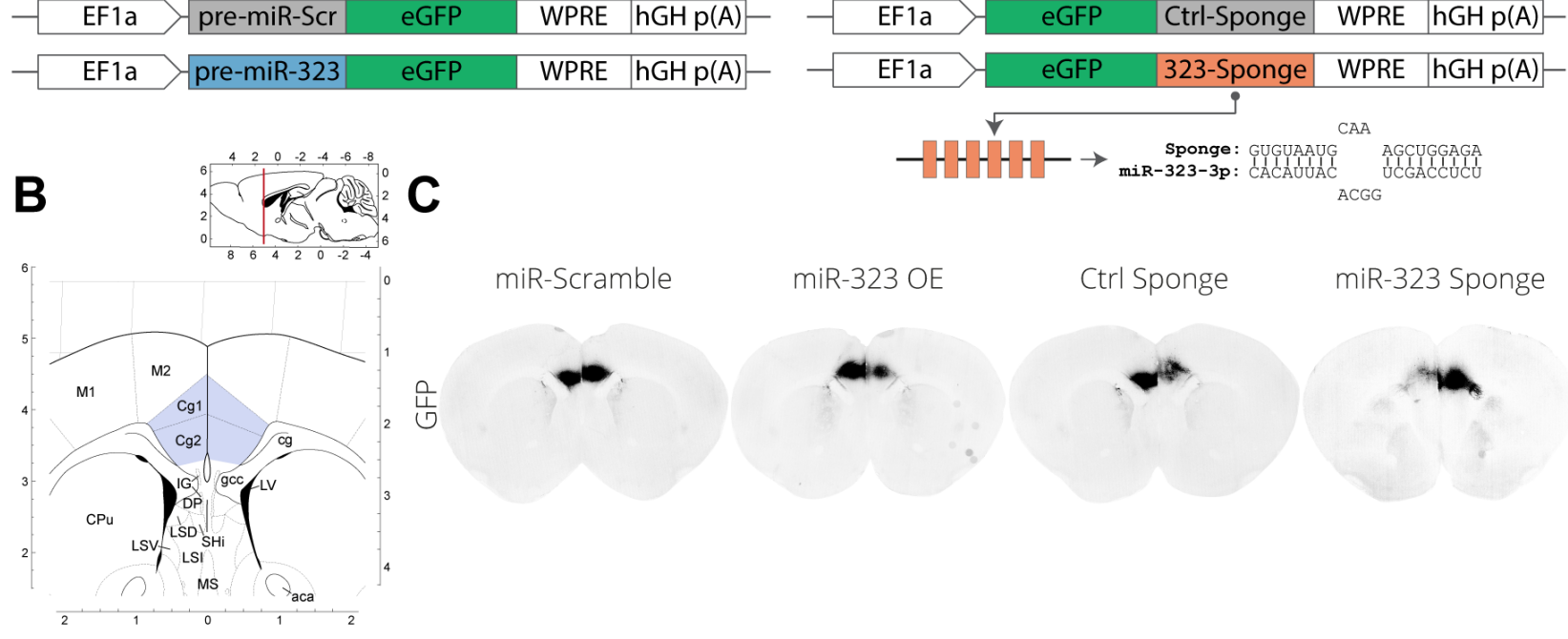

D 1. Open field (OF) 2. Elevated plus maze 3. Tail suspension

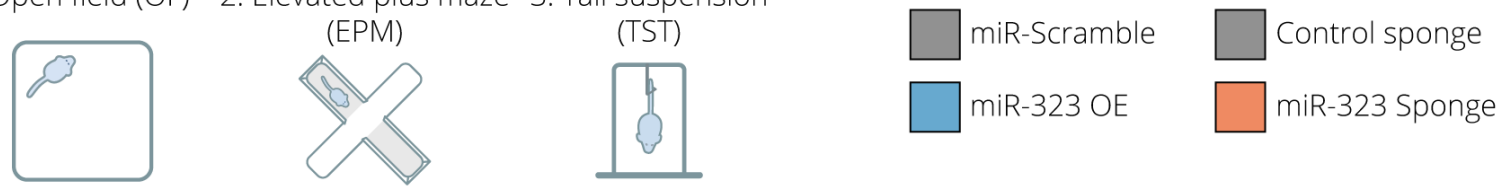

\section{E}
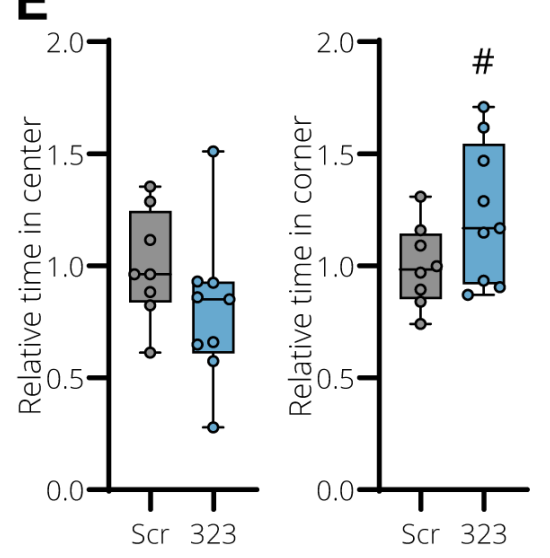

G
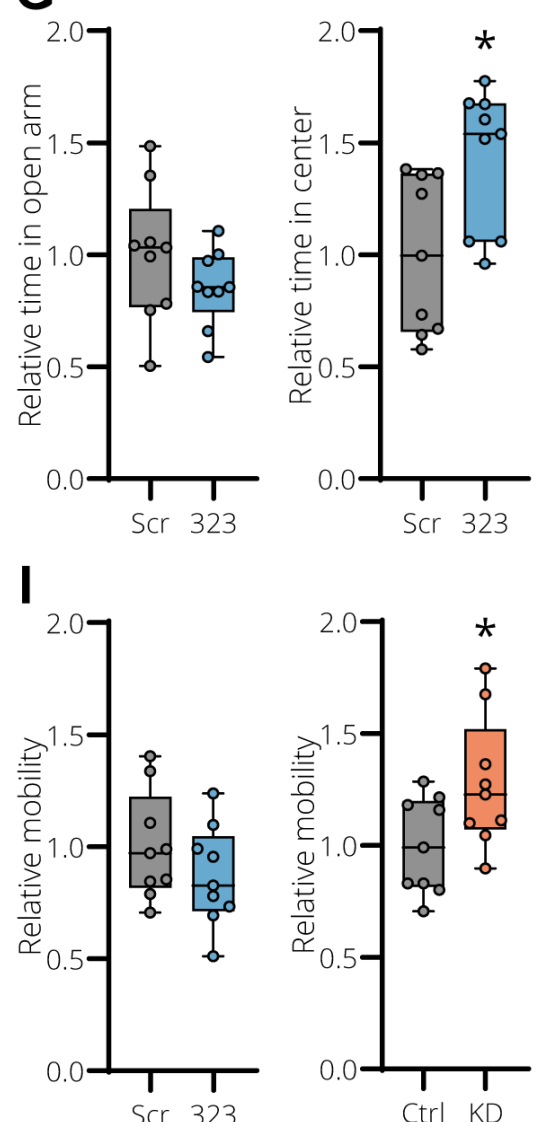

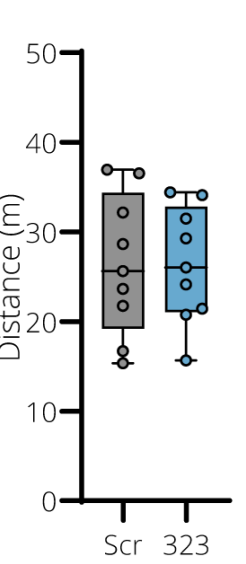

$F$
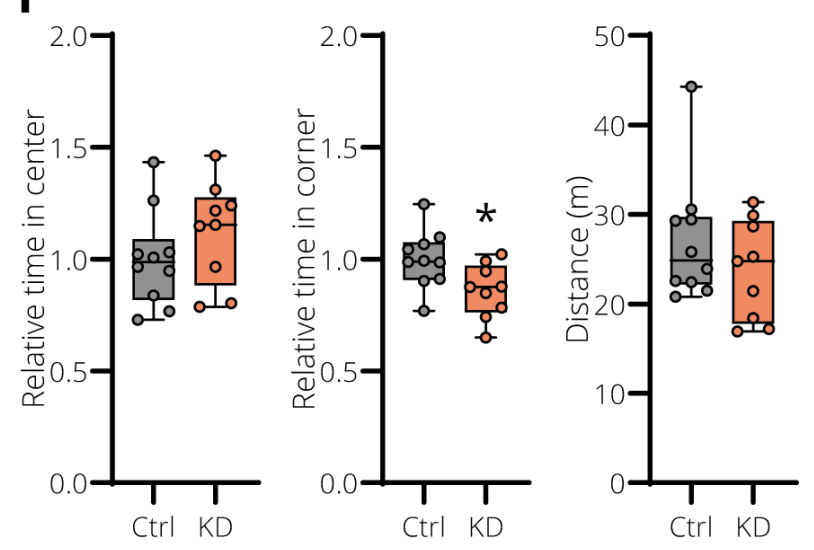

\section{H}
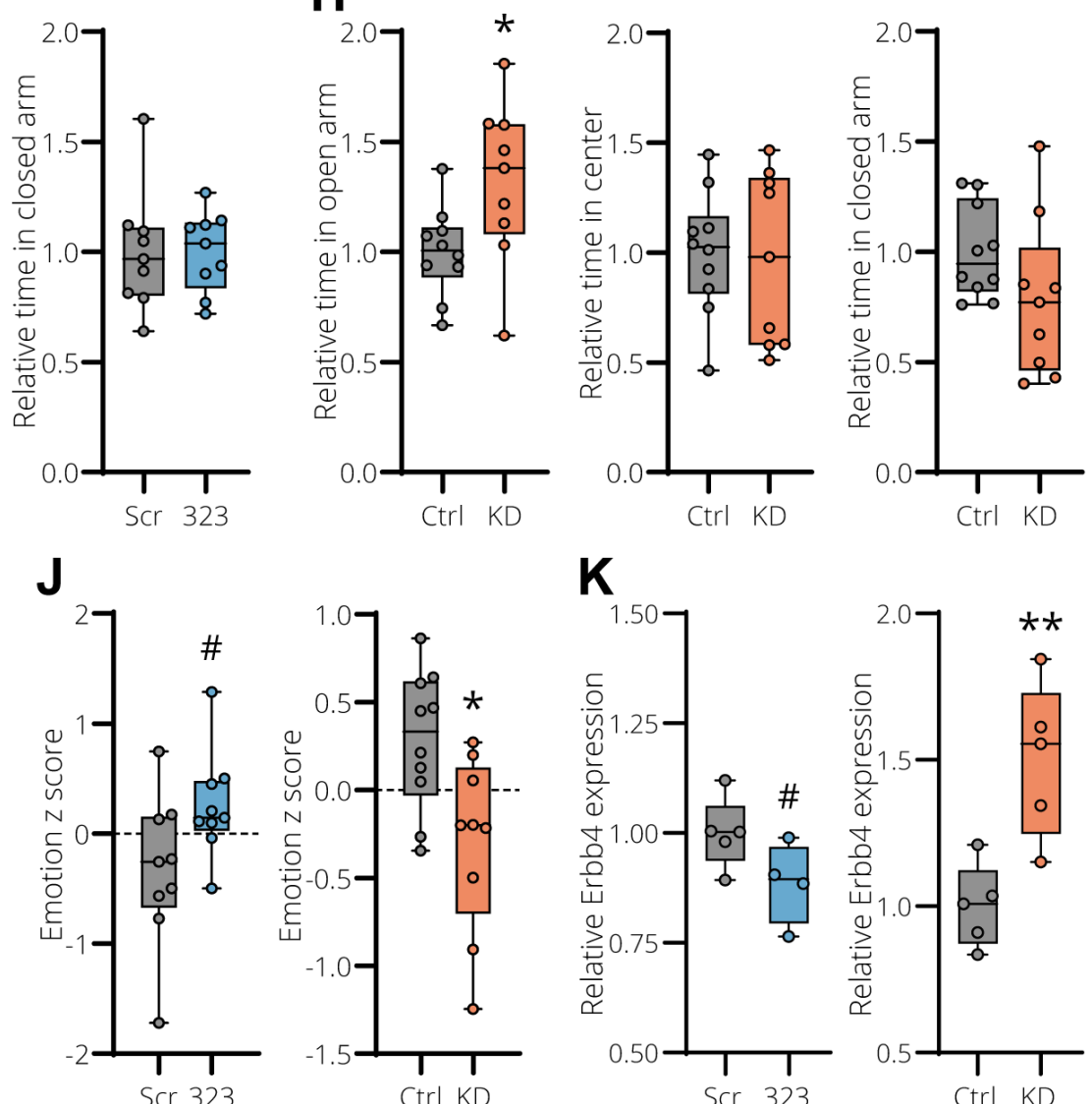

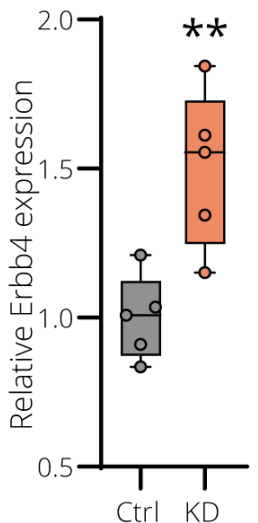


hsa-miR-323a-3p: CACAUUACACGGUCGACCUCU

mmu-miR-323-3p: CACAUUACACGGUCGACCUCU

\section{Region 1 binding site}

Position: 6425

Target: hsa-ERBB4 5' U

U UUUUCCCU

A 3'

$\begin{array}{ll}\text { GGGGGUUG CC } & \text { UAAUGUG } \\ \text { IIIIIII I I I } & \text { IIIIIII } \\ \text { UCUCCAGC GG } & \text { AUUACAC }\end{array}$

hsa-miR-323a-3p $\quad 3^{\prime} \quad$ U CAC

$5^{\prime}$

Position: 6351

Target: mmu-ERBB4 5' G

U U UUUUCCU

$\begin{array}{ll}\text { AGGGGUUG G CC } & \text { UAAUGUG } \\ \text { IIIIIII } 1 \text { I I I } & \text { I I I I I } \\ \text { UCUCCAGC U GG } & \text { AUUACAC }\end{array}$

mmu-miR-323-3p 3' $\quad 3^{\prime}$ U CAC

$5^{\prime}$

\section{Region 2 binding site:}

Position: 7459

Target: hsa-ERBB4 5' C AUU GU G U 3'

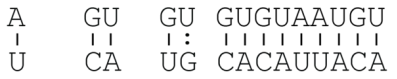

hsa-miR-323a-3p 3' CUC GC G $\quad$ C 5'

Position: 7400

Target: mmu-ERBB4 5' C AUU GU G A U 3'

A GU GU GUG AAUGU

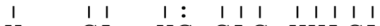

U CA UG CAC UUACA

mmu-miR-323-3p $\quad 3^{\prime} \quad$ CUC $\quad$ GC $\quad$ G $\quad$ A $\quad$ C 5' 